\title{
BMJ
}

\section{The economy-wide impact of pandemic influenza on the UK: a computable general equilibrium modelling experiment}

\author{
Richard D Smith, professor of health system economics, ${ }^{1}$ Marcus R Keogh-Brown, research fellow in \\ economic modelling, ${ }^{1}$ Tony Barnett, professorial research fellow and honorary professor, ${ }^{1,2}$ Joyce Tait, \\ professor and scientific adviser ${ }^{3}$
}

${ }^{1}$ Health Policy Unit, Department of Public Health and Policy, London School of Hygiene and Tropical Medicine, London WC1E 7HT

2London School of Economics and Political Science, London WC2A 2AE

${ }^{3}$ ESRC Innogen Centre, University of Edinburgh, Edinburgh EH1 1LZ Correspondence to: R D Smith Richard.Smith@lshtm.ac.uk

Cite this as: BMJ 2009;339:b457 doi:10.1136/bmj.b4571

\section{ABSTRACT}

Objectives To estimate the potential economic impact of pandemic influenza, associated behavioural responses, school closures, and vaccination on the United Kingdom. Design A computable general equilibrium model of the UK economy was specified for various combinations of mortality and morbidity from pandemic influenza, vaccine efficacy, school closures, and prophylactic absenteeism using published data.

Setting The 2004 UK economy (the most up to date available with suitable economic data).

Main outcome measures The economic impact of various scenarios with different pandemic severity, vaccination, school closure, and prophylactic absenteeism specified in terms of gross domestic product, output from different economic sectors, and equivalent variation.

Results The costs related to illness alone ranged between $0.5 \%$ and $1.0 \%$ of gross domestic product $(£ 8.4 \mathrm{bn}$ to $£ 16.8 \mathrm{bn}$ ) for low fatality scenarios, $3.3 \%$ and $4.3 \%$ ( $55.5 \mathrm{bn}$ to $£ 72.3 \mathrm{bn}$ ) for high fatality scenarios, and larger still for an extreme pandemic. School closure increases the economic impact, particularly for mild pandemics. If widespread behavioural change takes place and there is large scale prophylactic absence from work, the economic impact would be notably increased with few health benefits. Vaccination with a pre-pandemic vaccine could save $0.13 \%$ to $2.3 \%$ of gross domestic product ( $£ 2.2 \mathrm{bn}$ to $£ 38.6 \mathrm{bn}$ ); a single dose of a matched vaccine could save $0.3 \%$ to $4.3 \%$ ( $£ 5.0 \mathrm{bn}$ to $£ 72.3 \mathrm{bn}$ ); and two doses of a matched vaccine could limit the overall economic impact to about $1 \%$ of gross domestic product for all disease scenarios.

Conclusion Balancing school closure against "business as usual" and obtaining sufficient stocks of effective vaccine are more important factors in determining the economic impact of an influenza pandemic than is the disease itself. Prophylactic absence from work in response to fear of infection can add considerably to the economic impact.

\section{INTRODUCTION}

In the past century there were three major influenza pandemics $\left(1918,1957\right.$, and 1968-9). ${ }^{1}$ This century has seen an outbreak of severe acute respiratory syndrome (2003), H1N1 subtype of the influenza A virus (2009), and sporadic outbreaks of H5N1 influenza subtype. ${ }^{2}$ In addition to the direct health impacts of a serious outbreak, we should be concerned about the economic impact; especially at a time of global recession. ${ }^{3}$ Preparedness planning for a pandemic must therefore balance two key policy strands - maintaining "business as usual" to minimise the economic impact of a pandemic, and encouraging "social distancing" to minimise the health related impact of a pandemic ${ }^{4}$-as well as using resources such as antivirals and vaccinations.

This paper considers the tension inherent in these two policy strands. It provides evidence of the economy-wide impact of each approach, as well as the impact that vaccine development may have in reconciling the two objectives of minimising both the health and economic effects of a pandemic. A key consideration in this analysis is the role of public perception and confidence, expressed by "prophylactic absenteeism," where healthy people avoid social contact, including going to work. This response is likely to emerge at higher case fatality rates and to be moderated by the availability of effective vaccines (the current strain of H1N1 influenza seems to be highly infectious but not very deadly, and this may explain its limited economic impact to date).

\section{METHODS}

The analysis is based on a computable general equilibrium model of the $\mathrm{UK}^{5}$ over one year. The economy is specified in terms of several agents, including households, producers, and government, and based on data (in the form of a social accounting matrix, which represents income and expenditure in the economy by sector) for 2004 taken from the Global Trade Analysis Database $^{6}$ and national statistics. ${ }^{78}$ Computable general equilibrium modelling is described in further detail by Dervis et al. ${ }^{9}$

The economic impact of influenza in our model is assumed to occur through the labour supply, since illness and death cause both a reduction in the availability of labour and in its quality. Mitigation actions can also affect the labour supply by $(a)$ reducing labour 
when people are kept away from the workplace to avoid infection or $(b)$ by increasing labour supply compared with non-mitigated pandemic scenarios by reducing the number of infections and deaths ${ }^{10}$ and reducing the extent to which people feel the need to engage in prophylactic absenteeism.

\section{Pandemic impact}

Pandemic planning documents ${ }^{4112}$ anticipate clinical attack rates between $25 \%$ and $35 \%$, with a maximum of $50 \%$. We therefore use these three values in our disease scenarios. Based on previous pandemics, predicted case fatality rates for the UK range from $0.2 \%$ to $2.5 \%,{ }^{412} 13$ and the summary estimate for European pandemic preparedness plans is $0.37 \% .{ }^{11} \mathrm{We}$ used $0.4 \%$ as our base disease scenario and $2.5 \%$ for our severe scenario, with an extreme scenario of $10 \%$ based on severe acute respiratory syndrome (SARS). ${ }^{1314}$ We therefore have nine possible combinations of clinical attack rate and case fatality rate.

While deaths permanently remove labour from the workforce, absenteeism represents temporary removal. Illness absence will result in subsequent immunity to the virus, whereas those undertaking prophylactic absenteeism will still be vulnerable to infection. The Commission of the European Communities suggests that the duration of pandemic influenza illness is five to eight working days, ${ }^{11}$ and absence for seasonal flu is approximately five days. ${ }^{15}$ We therefore assume five days of illness for our mild scenario, seven days for severe, and 22 days for the extreme scenario, which is

\begin{abstract}
Glossary of terms
Computable general equilibrium model—A mathematical model of the whole economy that includes the cost minimising and profit maximising behaviour of producers, the consumption and saving behaviour of households and government, taxation mechanisms, and the use of labour, capital, and other factors in order to produce goods for investment or consumption. The model produces a benchmark solution which is then compared with alternative solutions incorporating policy change or other events simulated by the model. Counterfactual solutions can be compared with the benchmark solution to estimate the economic impact of the simulated policy or event.
\end{abstract}

Social accounting matrix-A matrix that represents the balanced income and expenditure flows of a regional, national, or global economy aggregated to make them a manageable size for use in a computable general equilibrium model. (The matrix rows represent income to the economy and the columns represent expenditure.)

Global trade model—A computable general equilibrium model of the global economy. Prophylactic absenteeism—Absence from work of a healthy individual in order to avoid infection.

Clinical attack rate-The percentage of individuals in a population who become infected.

Case fatality rate-The percentage of infected individuals who die.

Mortality rate-Percentage of individuals in a total population who die (clinical attack rate $\times$ case fatality rate).

Reactive school closure-Government closure of a school to reduce infection when a (government defined) proportion of children or staff is experiencing illness.

School closure associated with prophylactic absenteeism-Closure of schools caused by the amount of prophylactic absence by staff.

Transition point-The point at which the severity of the pandemic provokes sufficient fear to invoke a sudden increase in prophylactic absenteeism within the population. based on hospitalisation rates for SARS. ${ }^{16}$ All absences are estimated as a percentage of time lost from a working year of 220 days.

\section{Pandemic mitigation: vaccination}

Although the US recently announced that it expects to go from vaccine trial to mass vaccination within two months, ${ }^{18}$ and the UK has signed agreements (with GSK and Baxter) to purchase 132 million doses of pandemic-specific vaccine, ${ }^{19}$ specific vaccines are unlikely to be available for the first wave of infection. ${ }^{20}$ During this stage, pre-pandemic vaccines, based on existing virus strains, will be the only option for protection, giving approximately $20 \%$ efficacy and, when combined with other clinical countermeasures, reducing the pandemic's impact to that of seasonal influenza. ${ }^{21}$ Once matched vaccines become available they are likely to have $70-80 \%$ efficacy, probably requiring two doses at an interval of three weeks. Vaccine shelf life is currently about one year. ${ }^{22}$

We assumed two vaccination strategies - a pre-pandemic vaccine with $20 \%$ efficacy and a matched vaccine with 40\% efficacy (single dose) and 80\% efficacy (double dose). ${ }^{23}$ For all vaccines we assumed sufficient stocks for $60 \%$ coverage. Vaccination would have two potential impacts on a pandemic, reducing the number of infected individuals and moderating the extent of prophylactic absenteeism because people feel protected from infection.

\section{Pandemic mitigation: school closure}

School closures are believed to reduce the impact of the pandemic, since infection rates among children are high, ${ }^{4}$ and this is mentioned in many pandemic planning documents. ${ }^{124-26}$ Although we witnessed closure at the early stages of the H1N1 influenza pandemic, it has been suggested that closure later, when the epidemic is better established, will be more effective in delaying spread, but also inevitable if large sectors of the population adopt prophylactic absenteeism in the face of increasing reports of deaths. ${ }^{27}$ It is therefore important to distinguish between school closure as a reactive policy to a pandemic and school closure associated with prophylactic absenteeism.

Ferguson et al $^{10}$ suggest that reactive school closure will result in closure for $95 \%$ of the 15 weeks of the pandemic, regardless of how often they reopen (duration of school closure associated with prophylactic absenteeism cannot, of course, be known). Previous studies $^{2829}$ have assumed school closure at the four week peak of the pandemic, allowing for some variation around the two or three week disease peak cited in the Department of Health's pandemic plan. ${ }^{4}$ Any school closure policy will result in disruption for working parents and, based on peak pandemic duration and Ferguson's estimates, we present scenarios with four weeks and 14.25 weeks of school closure. We also consider the mitigation impact of school closure, which is estimated as $2 \%$ for a $34 \%$ clinical attack rate in the Ferguson paper and up to a maximum of between 
$13 \%$ and $17 \%$ in the paper by Cauchemez et al, ${ }^{30}$ which we approximate as $15 \%$.

The UK Labour Force Survey (2005) suggests that 25245000 individuals aged 16-64 are in paid employment, of whom 3900000 are women who have dependent children in the household. That is, $15.5 \%$ of the workforce comprises women who are probably responsible for dependent children. ${ }^{31} \mathrm{~A}$ small proportion of working men is also reported to be responsible for dependent children, ${ }^{32}$ bringing potential absenteeism estimates through school closure to $16.1 \%$. However, we made some attempt to correct this estimate to account for informal care by grandparents, working from home, etc. In addition, we assumed that those $54 \%$ of working parents who maintain working hours during school closure because of informal care ${ }^{32}$ will lose working hours equivalent to one person's illness duration when their informal caregiver is ill.

\section{Pandemic mitigation: prophylactic absenteeism}

Previous studies ${ }^{28} 2933$ have shown that a main driver of economic impact is behavioural change. Behavioural change can include changes in consumption patterns and prophylactic absence from work to avoid infection. Prophylactic absence from work is likely to be governed by personal choice related to fear and therefore is unlikely to be proportionate. There will be a transition point when the number of individuals who decide to take relatively drastic social distancing action to avoid infection increases rapidly over a short space of time.

We suggest that the transition point in public behaviour related to an influenza pandemic is likely to be heavily influenced by the case fatality rate but to be reasonably independent of the clinical attack rate since illness, by itself, causes limited fear if a full recovery is anticipated. The public response to the current H1N1 pandemic supports this.

The level of the case fatality rate at which such a transition point will occur is likely to be related to the density of deaths in "effective social networks"- that is, the prospect of death becomes rapidly personalised with the death of a member of one's network of relatives, friends, colleagues, and acquaintances. To our knowledge, no study has been conducted to determine the impact of prophylactic absenteeism on modelling predictions related to disease and economic impacts. Accurate estimation of this transition point for pandemic influenza would require extensive survey work, and it would be useful to undertake such research in future so as to build up a body of knowledge relevant to the kind of modelling reported here. In its absence we have selected the conservative value of about 300 people as the effective social network, to include close contacts such as family and friends (10 12 people), acquaintances, and work colleagues. ${ }^{34-38}$ On this basis almost everybody in the population will know someone who has died once the mortality rate reaches one death per 300 people, triggering prophylactic absenteeism.
Simple arithmetic suggests that there are 200000 discrete effective social networks in the UK $(60000000 /$ 300). However, many of these will overlap, and so the real figure for the number of discrete social networks involved in communication of news of a death, taking into account Facebook, texting, and other networking tools, is likely to be smaller. In the absence of research on this topic, a case fatality rate in the range 2.5-5\% seems to be a valid assumption for the expected transition point.

Not all individuals will avoid work during a pandemic, but a survey conducted after the SARS outbreak $^{31}$ indicated that about 34\% of the working population in Europe would be willing to take prophylactic absence from work in the event of an infectious disease outbreak. Although survey responses do not always reflect true behavioural responses, it is reasonable to assume that the $34 \%$ of respondents who reported themselves as willing, in theory, to avoid work for a serious pandemic, would do so at the transition point presented above.

It is difficult to predict the duration of such absenteeism, as high levels of fear might cause prolonged periods of absence by some. However, it might reasonably be assumed that in most cases, absentees would be forced to take annual leave (as longer term sick leave usually requires a doctor's authorisation). On this assumption, absenteeism is likely to last up to four weeks, and, since the peak of the pandemic is likely to last two or three weeks ${ }^{4}$ and the transition point is unlikely to be reached before the peak, this is presumed to be a reasonable upper limit.

Table 1 provides a summary of the assumed parameter estimates used in our disease scenarios together with the sources of these estimates.

\section{RESULTS}

\section{Model of economic impact of pandemic influenza}

The accuracy of these results is subject to the scenarios we have outlined, the model specification, and the economic data from 2004 underlying the model. Figure 1 shows the impact of various disease and mitigation scenarios on gross domestic product: disease only (with no mitigation), disease with four weeks of school closure, disease with pre-pandemic vaccine, disease with matched vaccine (single dose), and disease with matched vaccine (double dose). Each scenario is plotted for low, medium, and high clinical attack rate (25\%, 35\%, and 50\%) and low, high, and extreme case fatality rates $(0.4 \%, 2.5 \%$, and $10 \%)$, although both rates are adjusted to allow for mitigation effects in the mitigation scenarios.

\section{Low case fatality rate}

The first three histogram bars show the impact of a low case fatality rate, in which variations in clinical attack rate have little impact (loss of $0.51-1.02 \%$ of gross domestic product). However, the impact of four weeks of school closure is large, doubling or even tripling the impact of the disease alone. The results also show that in a low fatality pandemic a pre-pandemic 
Table 1|Parameter assumptions and their sources used to estimate effects of pandemic influenza and possible responses to it

\begin{tabular}{|c|c|c|}
\hline Parameter & Estimates & Evidence \\
\hline Clinical attack rate & 25\% (mild); 35\% (moderate); 50\% (high) & $\begin{array}{l}\text { Department of Health }(2007)^{4} \text {; Commission of the European Communities }(2005)^{11} \text {; } \\
\text { Health Protection Agency }(2006)^{12}\end{array}$ \\
\hline Case fatality rate & $0.4 \%$ (low); $2.5 \%$ (high); $10 \%$ (extreme) & Department of Health $(2008)^{13}$; Stadler et al $(2003)^{14}$ \\
\hline Duration of illness (working days) & 5 (low); 7 (high); 22 (extreme) & $\begin{array}{l}\text { Commission of the European Communities }(2005)^{11} \text {; Postma et al }(2005)^{15} \text {; Peiris et al } \\
(2003)^{16} \text {; McLean et al }(2005)^{17}\end{array}$ \\
\hline No of working days per year & 220 & $\begin{array}{l}\text { Assumes } 104 \text { days are weekends, } 8 \text { days are bank holidays, and } 33 \text { days of annual } \\
\text { leave or other absence }\end{array}$ \\
\hline Pre-pandemic vaccine efficacy & $20 \%$ & Department of Health $(2007)^{21}$ \\
\hline Matched vaccine efficacy & $40 \%$ (single dose); $80 \%$ (double dose) & Leroux-Roels et al $(2008)^{23}$ \\
\hline & 4 weeks & Based on duration of peak of pandemic, Department of Health $(2007)^{4}$ \\
\hline School closure duration & 14.25 weeks ( $95 \%$ of 15 week duration) & $\begin{array}{l}15 \text { weeks based on duration of pandemic, Department of Health }(2007)^{4} ; 95 \% \text { of } \\
\text { duration based on Ferguson et al }(2006)^{10}\end{array}$ \\
\hline $\begin{array}{l}\text { Percentage of parents needing to take time } \\
\text { off work if schools were closed }\end{array}$ & $16.1 \%$ & Sadique et al $(2008)^{33}$ \\
\hline $\begin{array}{l}\text { Percentage of parents with access } \\
\text { to informal care }\end{array}$ & $54 \%$ & Sadique et al (2008) $)^{33}$ \\
\hline Density of effective social networks & 300 people & Cheng et al $(2004)^{34}$; Chombart de Lauwe et al $(1953)^{35}$; Ko et al $(2006)^{36}$ \\
\hline $\begin{array}{l}\text { Percentage of population willing to take } \\
\text { prophylactic absence at the transition point }\end{array}$ & $34 \%$ & Sadique et al $(2007)^{31}$ \\
\hline Duration of prophylactic absence & 4 weeks & $\begin{array}{l}\text { Based on limitations of annual leave and duration of peak pandemic, Department of } \\
\text { Health }(2007)^{4}\end{array}$ \\
\hline $\begin{array}{l}\text { Transition point when prophylactic absence } \\
\text { becomes widespread }\end{array}$ & When $\geq 1$ death occurs in every social network* & See density of effective social networks \\
\hline \multirow{2}{*}{ Mitigation impact of school closures } & $\begin{array}{l}\text { Reduction in clinical attack rate equivalent to } 2 \% \text { in } \\
\text { a pandemic with } 34 \% \text { clinical attack rate }\end{array}$ & Ferguson et al $(2006)^{10}$ \\
\hline & $15 \%$ reduction in CAR & Cauchemez et al (2009) ${ }^{30}$ \\
\hline
\end{tabular}

*When mortality rate (clinical attack rate $\times$ case fatality rate) is $\geq 1 / 300$.

vaccine might result in savings of $0.13-0.26 \%$ of gross domestic product, and a matched vaccine could result in savings of $0.26-0.51 \%$ for a single dose and 0.49 $0.96 \%$ for a double dose. The transition point of prophylactic absenteeism is not reached in any of the scenarios with a low case fatality rate.

\section{High case fatality rate}

With a high case fatality rate, however, the transition point is reached (mortality rate becomes similar to that of the 1918 pandemic), so that individual change in behaviour to avoid infection yields large impacts of

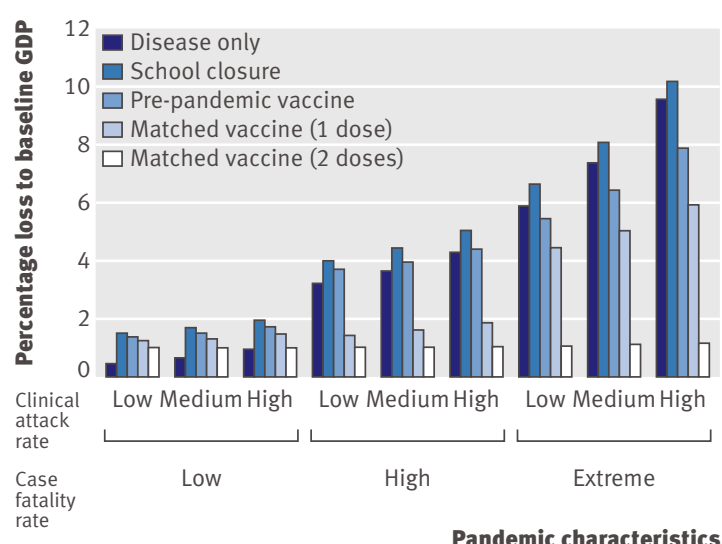

Fig 1| Effect of pandemic influenza on UK gross domestic product (GDP) according to various disease and mitigation scenarios (all vaccination strategies assumed to have $60 \%$ coverage)
$3.3 \%, 3.7 \%$, and $4.3 \%$ of gross domestic product for the low, medium, and high infection rates, respectively-emphasising that the mortality rate can, in such circumstances, be a more important determinant of economic impact than the infection rate.

The introduction of school closures in high fatality scenarios has less impact than in low fatality scenarios, with an additional impact of $0.75-0.8 \%$ of gross domestic product. A pre-pandemic vaccine would be insufficient to avoid the transition point in a high fatality pandemic and so would reduce the impact on gross domestic product by only $0.33-0.64 \%$. A matched vaccine, even if only single dose, would have sufficient effect to avoid the transition point and could therefore result in savings of 2.6-3.2\% of gross domestic product (roughly equivalent to half of the impact of the financial crisis over the past year (www.statistics.gov.uk/instantfi gures.asp)). Two doses of a matched vaccine are likely to reduce the impact further, yielding savings of 3-4\%.

\section{Extreme case fatality rate}

The extreme fatality scenarios predictably yield the largest impacts. Our assumptions dictate that for such a serious pandemic the transition point would be passed, and the low, medium, and high infection scenarios yield reductions in gross domestic product of $6.0 \%, 7.4 \%$, and $9.6 \%$, respectively. School closure increases this impact by $0.63-0.77 \%$, which is smaller than for the less severe scenarios as the mitigation impact of school closure reduces the severity of the disease. Pre-pandemic vaccine has some effect on reducing the impact of the 
school closure scenarios by 1.2-2.3\%, and a single dose of matched vaccine yields slightly larger savings of 2.2$4.3 \%$. The matched vaccine with two doses is the only mitigation strategy that avoids the transition to prophylactic absenteeism in the extreme fatality scenarios, reducing the overall impact of the pandemic to 1.1$1.2 \%$ of gross domestic product (much less than the impact of the current financial crisis).

\section{Alternative scenarios}

Alternatives to these scenarios have also been modelled but are not reported in detail here. In brief, schools closing for about $95 \%$ of the 15 weeks of the pandemic's duration and assuming a mitigation equivalent to $2 \%$ if the clinical attack rate was $34 \%$, as outlined by Ferguson et al, ${ }^{10}$ produces a $2.5 \%$ further reduction in gross domestic product compared with our four week closure scenarios; it reduces the infection rates, but the dominance of the case fatality rate in determining the transition point is such that the degree of prophylactic absenteeism remains the same.

Similarly, informal care by grandparents and friends, reducing the level of prophylactic absenteeism from $16.1 \%$ to $8.7 \%,{ }^{32}$ reduces the loss to gross domestic product by $0.56-0.58 \%$ in the four week school closure scenarios and by $1.8-2.0 \%$ in the longer closure scenario. Assuming the higher mitigation rate of $15 \%$ suggested by Cauchemez et al, ${ }^{30}$ which would apply to longer school closure scenarios, the severity of the economic impact of school closures is reduced in proportion to the severity of the pandemic. This reduction is quite small - up to $0.25 \%$ of gross domestic product for non-extreme scenarios and up to $0.9 \%$ of gross domestic product in the most extreme and unmitigated scenario - so overall reductions in gross domestic product are still between $1 \%$ and $2 \%$ larger than with the four week school closure scenarios. School closure's failure to mitigate the impact of the disease despite the assumption of its efficacy is due to the large amount of absenteeism induced by school closures, which, in our model, is not affected by the clinical attack rate.

Additional scenarios relating to the swine flu pandemic and alternative vaccine efficacy assumptions are included in an online appendix.

Table 2 |Equivalent variation as percentage of gross domestic product (GDP) and £bn for different influenza pandemic scenarios

\begin{tabular}{|c|c|c|c|c|c|}
\hline \multicolumn{2}{|c|}{ Pandemic scenario } & \multicolumn{2}{|c|}{ Disease only } & \multicolumn{2}{|c|}{4 week school closure } \\
\hline Case fatality rate & Clinical attack rate & $\%$ of GDP & $£ b n$ & $\%$ of GDP & $£ b n$ \\
\hline \multirow{3}{*}{ Low } & Low & -0.7 & -11.8 & -5.1 & -85.8 \\
\hline & Medium & -1 & -16.8 & -5.4 & -90.8 \\
\hline & High & -1.5 & -25.2 & -5.8 & -97.6 \\
\hline \multirow{3}{*}{ High } & Low & -4.8 & -80.7 & -8.8 & -148.0 \\
\hline & Medium & -5.4 & -90.8 & -9.4 & -158.1 \\
\hline & High & -6.3 & -106.0 & -10.3 & -173.2 \\
\hline \multirow{3}{*}{ Extreme } & Low & -8.7 & -146.3 & -12.7 & -213.6 \\
\hline & Medium & -10.8 & -181.7 & -14.8 & -248.9 \\
\hline & High & -14 & -235.5 & -17.9 & -301.1 \\
\hline
\end{tabular}

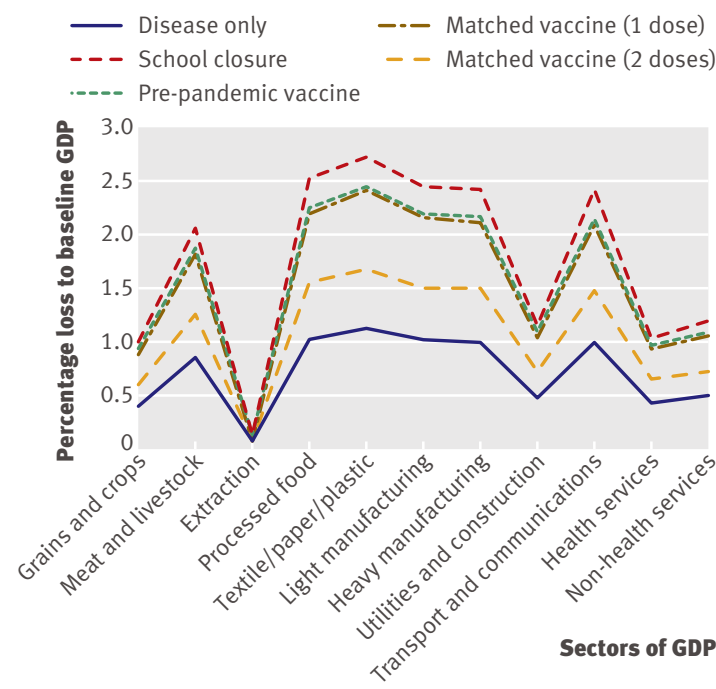

Fig 2 | Impact of pandemic influenza on different economic sectors of UK gross domestic product (GDP)

\section{Impact of pandemic on different economic sectors}

Figure 2 shows the impact on different sectors of the economy for the $35 \%$ clinical attack rate and $0.4 \%$ case fatality rate scenario. The pattern is similar across scenarios. The lowest impacts are seen in the extraction sector (mining, quarrying, forestry and fishing) followed by crops, utilities, and health and non-health services. The largest impacts are in the meat and livestock, processed foods, textiles/paper/plastics, manufacturing, and transport and communications sectors.

\section{Equivalent variation}

Computable general equilibrium modelling also produces the welfare measure of equivalent variation, which represents the amount of money that, if an economic change does not happen, leaves the population just as well off as if the change had occurred. This may be thought of as the amount of money that the population might be willing to pay to avert the pandemic. For the purposes of this paper, the welfare measure is quoted as a percentage of gross domestic product, with the results presented in table 2 . In order to avoid the economic impact of the pandemic, the cost that the population might be willing to pay ranges from $0.7 \%$ ( $111.8 \mathrm{bn})$ for the mildest disease-only scenario to $14 \%$ (£235bn) for the most extreme. School closure increases these values to $5.1 \%$ ( $£ 85.8 \mathrm{bn})$ in the mildest scenario to $17.9 \%$ ( $£ 301.1 \mathrm{bn}$ ) for the most severe scenario.

\section{DISCUSSION}

Our results show that, depending on the disease severity, pandemic influenza alone could reduce gross domestic product by $0.5-4.3 \%$. Extending fatality rates beyond those observed in previous pandemics to a SARS-like case fatality rate of $10 \%$ yields an impact of $5.9-9.6 \%$ of gross domestic product ( $£ 99.2 \mathrm{bn}$ to $£ 161.5 \mathrm{bn}$ ). School closure, and its related absenteeism, causes a notable increase in economic loss, and caution 


\section{WHAT IS ALREADY KNOWN ON THIS TOPIC}

Fear induced behavioural changes or government sanctioned absences from work or school in response to a flu pandemic could have a substantial economic impact, and these losses may not be balanced by large health benefits

\section{WHAT THIS PAPER ADDS}

Vaccines play a major role in mitigating the economic impact of a pandemic regardless of the characteristics of the disease

If increased fear caused by deaths within an individual's social network provokes prophylactic absence from work, large economic loss could result

might therefore be advised in pursuing this policy when the case fatality rate is low.

A pre-pandemic vaccine with moderate efficacy and $60 \%$ coverage could result in large relative savings for any low or high fatality pandemic of $£ 2.2 \mathrm{bn}-£ 10.8 \mathrm{bn}$, or $£ 20.2 \mathrm{bn}-£ 39.0 \mathrm{bn}$ for extreme scenarios. A matched vaccine, even if only available in sufficient time for a single dose to be administered to $60 \%$ of the population, would result in substantially higher savings, in both low and high fatality scenarios, and a double dose of a matched vaccine could keep the loss in gross domestic product to below $3 \%$, even with the most extreme pandemic and long school closureswhich is little more than half the impact of the current recession, although recovery from a severe flu event would probably be much more rapid. Estimates suggest that the planned $\mathrm{H} 1 \mathrm{~N} 1$ flu vaccination will cost about $£ 6$ per person (www.bloomberg.com/apps/ news?pid=20601124\&sid=auYQiQYJ.73I), yielding a total cost of about $£ 792 \mathrm{~m}$, compared with savings that start from $£ 2.2 \mathrm{bn}$ for the mildest pandemic. Our results suggest that, even in a mild pandemic, a vaccine costing $£ 16.60$ per person would be beneficial in terms of its health impact without imposing a burden on the economy.

Our results also consider the possibility that there is a transition point in case fatality rate, above which many individuals might change their usual behaviour and avoid work in an attempt to avoid infection. Evidence suggests that $50 \%$ prophylactic absence, which is larger than our assumed absence rates, is likely to reduce a $34 \%$ clinical attack rate by only $1 \%$ (case fatality rate is unchanged as this is dependent on the disease). ${ }^{10}$ Since these absence rates could cost the economy billions of pounds, prophylactic absence should be discouraged except in exceptional circumstances.

\section{Limitations and strengths of study}

This work does not take into account consumption effects from avoidance of public places, entertainment events, and changes in shopping patterns, although further work is under way to establish an evidence base from which these effects can be modelled. Impacts on trade, imports, and exports have not been examined here as it is difficult to assess these impacts accurately with a single-country model. A study with a global trade model is ongoing and will supplement the findings presented here.
The strength of our findings depends on the underlying assumptions which, though based on published evidence where possible, are subject to the bias of surveys, the unpredictability of the disease and its resultant impact on policies and behavioural change. We included estimates of school closure and prophylactic absence, but their true values in the middle of a pandemic could vary widely. However, this paper extends previous work ${ }^{24}$ by using the best available estimates to approximate the impact of social networks on behavioural change, considering various lengths of school closure, their feedback effects on the pandemic, and the impact of informal care both in mitigating absenteeism due to school closure and in causing absenteeism by parents when informal carers are unwell, as well as considering the impact of various vaccination strategies on disease and behaviour change.

\section{Conclusion}

Pandemic influenza itself, if it occurs within the bounds of severity outlined in pandemic plans, will not yield unprecedented economic impacts: even a high fatality pandemic with high levels of infection would reduce gross domestic product by less than 4.5\%. However, two factors will compound the disease's impact. Firstly, a pandemic in the near future would impose additional strain on an economy that is already stretched by recession, exaggerating the effect of recession and slowing economic recovery. Secondly, although the direct economic impact of disease is relatively small, school closures and prophylactic absenteeism, whether imposed by government or the result of fear of infection in the population, could greatly increase the economic impact.

In the event of a mild pandemic, long periods of school closures will not be necessary and could greatly multiply the economic impact of the disease and should therefore be minimised. In more serious pandemics, the relative economic impact of school closures decreases and the gains from school closure in mitigating the pandemic increase, so a policy of school closure should take into account the severity of the disease. However, such a policy should be limited in its duration-sufficient to maximise the lowering of peak disease levels and maintain a functioning health service, but allowing schools to open at other times. In an extreme pandemic, the relative incremental cost of school closure is small and should not influence policies that would minimise deaths.

Our "transition point" estimates provide an example of how fear induced behavioural change could greatly increase the economic impact of a pandemic while providing questionable health gains. We suggest that the overall mortality rate is the driver of this behavioural change, and vaccinations, whether pre-pandemic or matched vaccine, could be extremely important in preventing mortality rates from reaching the "transition point." The cost of vaccinations is likely to be less than the economic savings gained from vaccination, even in the mildest of pandemics, and in the event of a high or extreme fatality pandemic a matched vaccine 
might be the only method to avoid the unprecedented economic effects of behavioural change.

Contributors: RDS conceived the idea of a computable general equilibrium application for influenza, advised on the modelling and scenarios, and contributed to the drafting of the paper. MRK-B was responsible for the modelling, the underlying dataset, construction of modelling scenarios and shocks, and drafted the paper. TB and JT conceived the idea of the transition point based on social networking theory, advised on the scenarios and vaccination strategies, and contributed to the drafting of the paper. MRK-B is guarantor for the study. Funding: No specific funding for this study.

Competing interests: None declared.

Ethical approval: Not required for this study.

Data sharing: Model output data are available on request from Marcus. Keogh-Brown@lshtm.ac.uk

1 Macfarlane JT, Lim WS. Bird flu and pandemic flu. BMJ 2005;331:975-6.

2 Coker R. Swine flu. BMJ 2009;338:b1797.

3 Keogh-Brown MR, Smith RD. The economic impact of SARS: how does the reality match the predictions? Health Policy 2008;88:110-20.

4 Department of Health. Pandemic influenza: a national framework for responding to an influenza pandemic. DH, 2007.

5 Löfgren H, Harris RL, Robinson S. A standard computable general equilibrium (CGE) model in GAMS. International Food Policy Research Institute (IFPRI), 2001.

6 Narayanan B, Walmsley TL. Global trade, assistance, and production: the GTAP 7 data base. Center for Global Trade Analysis, Purdue University, 2008.

7 Fofona I, Lemelin A, Cockburn J. Balancing a social accounting matrix: theory and application. Centre Interuniversitaire sur le Risque les Politiques Economiques et L'Emploi (CIRPEE), 2005.

8 Arndt C, Robinson S, Tarp F. Parameter estimation for a computable general equilibrium model: a maximum entropy approach. Perdue University, IFPRI, University of Copenhagen, 1999. (Discussion paper No 40.)

9 Dervis K, de Malo J, Robinson S. General equilibrium models for development policy. Cambridge University Press, 1982.

10 Ferguson NM, Cummings DAT, Fraser C, Cajka JC, Cooley PC, Burke DS. Strategies for mitigating an influenza pandemic. Nature 2006;442:448-52

11 Commission of the European Communities. Communication from the commission to the council, the European parliament, the European economic and social committee and the committee of the regions on pandemic influenza preparedness and response planning in the European community. EC, 2005.

12 Health Protection Agency. Pandemic influenza contingency plan. HPA, 2006.

13 Department of Health. Scientific Pandemic Influenza Advisory Committee (SPI): subgroup on modelling, modelling summary. DH, 2008.

14 Stadler K, Masignani V, Eickmann M, Becker S, Abrignani S, Klenk H $\mathrm{D}$, et al. SARS-beginning to understand a new virus. Nat Rev Micro 2003;1:209-18.

15 Postma MJ, Jansema P, Sheijbeler HWKFH, van Genugten MLL. Scenarios on costs and savings of influenza treatment and prevention for Dutch healthy working adults. Vaccine 2005;23:5365-71.

16 Peiris JSM, Chu CM, Cheng VCC, Chan KS, Hung IFN, Poon LLM, et al. Clinical progression and viral load in a community outbreak of coronavirus-associated SARS pneumonia: a prospective study. Lancet 2003;361:1767-72.
17 McLean A, May R, Pattison J, Weiss R. SARS: a case study in emerging infections. Oxford University Press, 2005.

18 Butler D. US puts flu vaccines on trial. Nature News 2009;460:562 (www.nature.com/news/2009/090728/full/ 460562a.htms).

19 Department of Health. Agreements secured for pre-pandemic vaccine in the UK. DH, 2009 (www.dh.gov.uk/en/Publichealth/Flu/ Swineflu/DH_099248).

20 Ulmer JB, Valley U, Rappuoli R. Vaccine manufacturing: challenges and solutions. Nat Biotechnol 2006;24:1377-83.

21 Department of Health. Pre-pandemic and pandemic influenza vaccines: scientific evidence base. DH, 2007 (www.dh.gov.uk/en/ Publicationsandstatistics/Publications/ PublicationsPolicyAndGuidance/DH_077276).

22 European Centre for Disease Prevention and Control. Technical report: expert advisory groups on human $\mathrm{H} 5 \mathrm{~N} 1$ vaccines, scientific questions.ECDC, 2007.

23 Leroux-Roels I, Bernhard R, Gerard P, Drame M, Hanon E, Leroux-Roels G. Broad clade 2 cross-reactive immunity induced by an adjuvanted clade $1 \mathrm{rH} 5 \mathrm{~N} 1$ pandemic influenza vaccine. PLoS One 2008;3:e1665.

24 Department of Health. UK Health Departments' Influenza pandemic contingency plan. DH, 2005.

25 US Department of Health and Human Services. HHS pandemic influenza plan. HHS, 2005.

26 Public Health Agency of Canada. The Canadian pandemic influenza plan for the health sector. PHA, 2006.

27 Cauchemez S, Valleron A-J, Boelle P-Y, Flahault A, Ferguson NM. Estimating the impact of school closure on influenza transmission from Sentinel data. Nature 2008;452:750-4.

28 Keogh-Brown MR, Smith RD, Edmunds W, Beutels P. The macroeconomic impact of pandemic influenza: estimates from models of the UK, France, Belgium and the Netherlands. Eur J Health Econ (forthcoming).

29 Keogh-Brown MR, Wren-Lewis S, Edmunds WJ, Beutels P, Smith RD. The possible macroeconomic impact on the UK of an influenza pandemic. Health Econ [forthcoming].

30 Cauchemez S, Ferguson NM, Wachtel C, Tegnell A, Saour G, Duncan B, et al. Closure of schools during an influenza pandemic. Lancet Infect Dis 2009;9:473-81.

31 Sadique MZ, Edmunds WJ, Smith RD, Meerding WJ, de Zwart O, Brug J, et al. Precautionary behavior in response to perceived threat of pandemic influenza. Emerg Infect Dis 2007;13:1307-13.

32 Sadique MZ, Adams E, Edmunds W. Estimating the costs of school closure for mitigating an influenza pandemic. BMC Public Health 2008;8:135.

33 Keogh-Brown MR, Smith RD. The economic impact of SARS: how does the reality match the predictions. Health Policy 2008;88:110-20.

34 Cheng C, Tang C. The psychology behind the masks: psychological responses to the severe acute respiratory syndrome outbreak in different regions. Asian Journal of Social Psychology 2004;7:3-7.

35 Chombart de Lauwe PH, Antoine S, Bertin JLC, CouvreurCJG. Paris et l'agglomeration parisienne. Revue Française de Science Politique 1953;3(3):630-3.

36 Ko C, Yen C, Yen J, Yang M. Psychosocial impact among the public of the severe acute respiratory syndrome epidemic in Taiwan. Psychiatry Clin Neurosci 2006;60:297-403.

37 Wang $\mathrm{H}$, Wellman B. Social connectivity in America: changes in adult friendship network size from 2002 to 2007. American Behavioral Scientist [forthcoming].

38 Spencer L, Pahl R. Rethinking friendship: hidden solidarities today. Princeton University Press, 2006.

Accepted: 2 November 2009 